\title{
Identification and characterization of microRNAs and their targets in high-altitude stress-adaptive plant maca (Lepidium meyenii Walp)
}

\author{
Sujay Paul ${ }^{1,2}$
}

Received: 6 December 2016/ Accepted: 19 April 2017/Published online: 30 May 2017

(C) Springer-Verlag Berlin Heidelberg 2017

\begin{abstract}
MicroRNAs (miRNAs) are endogenous, short ( 21-nucleotide), non-coding RNA molecules that play pivotal roles in plant growth, development, and stress response signaling. In this study using recently published draft genome sequence of a high-altitude plant maca ( $L e$ pidium meyenii Walp) and applying genome-wide computational-based approaches, a total of 62 potentially conserved miRNAs belonging to 28 families were identified and four (lme-miR160a, lme-miR164c, lme-miR 166a, and lme-miR 319a) of them further validated by RT-PCR. Deploying psRNATarget tool a total of 99 potential miRNA target transcripts were also identified in maca. Targets include a number of transcription factors like Squamosa promoter-binding, NAC, MYB, auxin response factor, APETALA, WRKY, and F-box protein. To the best of my knowledge, this is the first genome-based miRNA profiling of a high-altitude plant.
\end{abstract}

Keywords Maca (Lepidium meyenii Walp) · High-altitude plant $\cdot$ Stress $\cdot$ miRNA $\cdot$ MFEI $\cdot$ miRNA targets

$\begin{array}{ll}\text { Abbreviations } \\ \text { miRNA } & \text { MicroRNA } \\ \text { MFE } & \text { Minimum folding free energy } \\ \text { MFEI } & \text { Minimum folding free energy index }\end{array}$

Sujay Paul

sujay.p07@gmail.com

1 Division of Plant Biology, Bose Institute, P 1/12 CIT Scheme VII M, Kolkata 700054, India

2 Present Address: Laboratorio de Micología y Biotecnología, Universidad Nacional Agraria La Molina, Av. La Molina s/n, 12 Lima, Peru

\section{Introduction}

Maca (Lepidium meyenii Walp), belonging to the Brassicaceae family, is an economically important plant cultivated in the central Andean region at $4000-4450 \mathrm{~m}$ above sea level. Its tremendous health benefits, particularly to reproduction and fertility, have drawn great investments from pharmacological research in recent years (Piacente et al. 2002; Shin et al. 2010). Maca roots contain several secondary metabolites of interest including glucosinolates, fatty acid esters, phytosterols, alkaloids, and alkamides (macamides) (Piacente et al. 2002). Due to its restricted cultivation area at high altitude, maca manifests strong endurance to extreme environmental conditions such as low temperatures in combination with high irradiance, strong winds, and oxidizing air pollutants.

MicroRNAs are endogenous, non-coding, small RNAs ranging in length from 20 to 24 nucleotides (Huang et al. 2016). Post-transcriptional gene regulation mediated by endogenous miRNAs play a crucial role in various aspects of plant development as well as adaptation to biotic and abiotic stresses (Naya et al. 2014; Khraiwesh et al. 2012; Paul et al. 2011; Kundu et al. 2017). In plants, mature miRNAs are generated from longer stem-loop RNA precursors (Pre-miRNA) with the aid of ribonuclease III-like Dicer (DCL1) enzyme (Fukudome and Fukuhara 2017). Despite the fact that miRNAs have a great role in stress responses, till date no scientific initiative has been taken to study maca miRNAs. Moreover, very few reports are available on medicinal plants for miRNA regulation of their bioactive compounds or secondary metabolites. With the recent draft genome sequence available (http://www. herbal-genome.cn/) (Zhang et al. 2016), it is important to exploit this information for better understanding the physiological processes in maca. 


\section{Materials and methods}

\section{Computational prediction of maca miRNAs}

A set of total of 811 mature Arabidopsis miRNAs (downloaded from miRbase 21) were BLASTn search against maca genome and sequences with exact match were chosen manually. It is well documented that during in silico miRNA prediction least number of allowed mismatches between putative miRNAs and known miRNAs produce more accurate results and that is why maca miRNAs which showed 0 mismatches with known Arabidopsis miRNAs were chosen in this study. The possible precursor (premiRNA) sequences of approximately 400-nt (200 nt upstream and $200 \mathrm{nt}$ downstream to the BLAST hit region) were extracted and sequences coded for proteins were removed. Stable secondary structures of the remaining precursor sequences were predicted using mfold web server (http://unafold.rna.albany.edu/?q=mfold/mfold-references) following previously described filtering criteria (Zhang et al. 2008) as follows: (1) the secondary structure of the precursor sequences should have the stem-loop structure that contains a mature miRNA sequence within one arm and no loop or break in the mature miRNA sequences; (2) the potential miRNA sequence should not be located on the terminal loop of the hairpin structure; (3) mature miRNAs should have fewer than nine mismatches with the opposite miRNA* sequence (Yang et al. 2010); and (4) the predicted stem-loop candidates should have higher MFEIs and negative minimum folding free energies. The formula for calculating MFEI is as follows:

MFEI $=\frac{(\text { MFE } / \text { length of RNA sequence }) \times 100}{\% \text { GC content }}$

\section{Analysis of miRNA expression}

For the experimental validation of some predicted maca miRNAs such as lme-miR160a, lme-miR164c, lme-miR 166a, and lme-miR 319a by RT-PCR (reverse transcription), small RNA was first isolated from maca leaves using mir Premier microRNA Isolation Kit (Sigma-Aldrich). $1 \mu \mathrm{g}$ of aforesaid maca small RNA was polyadenylated (using modified oligo dT primer) and reverse transcribed at $37{ }^{\circ} \mathrm{C}$ for $1 \mathrm{~h}$ in $10 \mu \mathrm{l}$ reaction mixture using Mir-X miRNA First-Strand Synthesis kit (Clontech). The obtained cDNA was then amplified by GeneAmp PCR system 2400 (Perkin Elmer) using entire predicted miRNA sequence as sense primer and adapter-specific mRQ $3^{\prime}$ primer provided with Mir-X miRNA qRT-PCR SYBR kit (Clontech) as antisense primer. $100 \mathrm{ng}$ cDNA was used as template for the PCR. The PCR was programed as follows: initial denaturation at $95{ }^{\circ} \mathrm{C}$ for $3 \mathrm{~min}$, followed by 35 cycles of denaturation at $94{ }^{\circ} \mathrm{C}$ for $30 \mathrm{~s}$, annealing at
$60{ }^{\circ} \mathrm{C}$ for $30 \mathrm{~s}$, extension at $72{ }^{\circ} \mathrm{C}$ for $25 \mathrm{~s}$, and a final elongation step at $72{ }^{\circ} \mathrm{C}$ for $7 \mathrm{~min}$. The resulting PCR products $(\sim 70 \mathrm{bp})$ were checked in $2 \%$ agarose gel with EtBr staining.

\section{Prediction of miRNA targets and their functional annotation}

The Plant Small RNA Target Analysis Server (psRNATarget) was used in this study to predict maca miRNA targets (http://plantgrn.noble.org/psRNATarget/). Due to non-availability of maca protein database in psRNATarget server target transcript search was performed against protein database of Arabidopsis thaliana. The following parameters were employed in prediction of miRNA targets in maca: (a) maximum exception of 3.0, length of complementarity score: 20. (b) Target accessibility-allowed maximum energy to unpair the target site (UPE): 25. (c) Flanking length around the target accessibility analysis: $17 \mathrm{bp}$ upstream and $13 \mathrm{bp}$ downstream. (d) Range of central mismatch leading to translation inhibition: 9-11 nt.

Gene ontology analysis of the identified target transcript was executed by AmiGo (http://amigo.geneontology.org/ amigo) and three important components such as biological process, cellular component, and molecular function associated with each GO term were inferred.

\section{Results and discussion}

\section{Characterization of maca miRNAs}

With high stringent filtering approach, a total of 62 potential conserved miRNAs belonging to 28 families were identified in maca (Table 1). Among them, 28 miRNAs ( $\sim 45 \%$ ) were located in $5^{\prime}$ arm of the precursor while 34 $(\sim 55 \%)$ located in $3^{\prime}$ arm suggesting that maca miRNAs are located in both the arms of the precursor void any preference. Precursors of maca miRNAs also showed great variability in their size ranging from 76 to 227 with an average of $117 \pm 33$ (Table 1) which represent good agreement with those reported for other plant species such as soybean, cotton, and maize (Zhang et al. 2008; Wang et al. 2011, 2012). lme-miR 2111b-3p showed the shortest precursor length of $76 \mathrm{nt}$ while vra lme-miR 169a showed the longest one of $227 \mathrm{nt}$. The MFEI is a useful criterion for distinguishing miRNAs from other types of coding or non-coding RNAs. In this study, the identified precursors have higher MFEI values (0.73-1.43) with an average of $1.00 \pm 14.0$ which is much higher than that of tRNAs (0.64), rRNAs (0.59), or mRNAs (0.62-0.66), respectively (Zhang et al. 2006). The secondary structure of the 
Table 1 Potential conserved miRNAs in maca

\begin{tabular}{|c|c|c|c|c|c|c|c|c|}
\hline Identified miRNAs & $\mathrm{LM}(\mathrm{nt})$ & miRNA sequences & Input sequence & Strand & Location & $\mathrm{LP}(\mathrm{nt})$ & MFEs $(\Delta G)$ & MFEI \\
\hline lme-miR156a & 20 & UGACAGAAGAGAGUGAGCAC & scaffold675 & $+/+$ & $5^{\prime}$ & 91 & -44.30 & 1.05 \\
\hline lme-miR156a-3p & 22 & GCUCACUGCUCUUUCUGUCAGA & scaffold568 & $+/+$ & $3^{\prime}$ & 128 & -47.90 & 0.87 \\
\hline lme-miR $156 b-3 p$ & 23 & UGCUCACCUCUCUUUCUGUCAGU & scaffold 236 & $+/+$ & $3^{\prime}$ & 101 & -45.50 & 0.97 \\
\hline lme-miR156c-3p & 22 & GCUCACUGCUCUAUCUGUCAGA & scaffold 675 & $+/+$ & $3^{\prime}$ & 86 & -43.80 & 1.07 \\
\hline lme-miR157a & 21 & UUGACAGAAGAUAGAGAGCAC & scaffold344 & $+/+$ & $5^{\prime}$ & 131 & -49.40 & 1.01 \\
\hline lme-miR157a-3p & 21 & GCUCUCUAGCCUUCUGUCAUC & scaffold78 & $+/+$ & $3^{\prime}$ & 86 & -44.40 & 1.35 \\
\hline lme-miR157c-3p & 21 & GCUCUCUAUACUUCUGUCACC & scaffold344 & $+/+$ & $3^{\prime}$ & 151 & -54.90 & 0.96 \\
\hline lme-miR159c & 21 & UUUGGAUUGAAGGGAGCUCCU & scaffold613 & $+/-$ & $3^{\prime}$ & 201 & -66.60 & 0.89 \\
\hline lme-miR160a & 21 & UGCCUGGCUCCCUGUAUGCCA & scaffold 249 & $+/+$ & $5^{\prime}$ & 81 & -45.70 & 1.04 \\
\hline lme-miR160a-3p & 21 & GCGUAUGAGGAGCCAUGCAUA & scaffold 249 & $+/+$ & $3^{\prime}$ & 96 & -48.70 & 0.99 \\
\hline lme-miR160c-3p & 21 & CGUACAAGGAGUCAAGCAUGA & scaffold 468 & $+/-$ & $3^{\prime}$ & 90 & -40.30 & 1.03 \\
\hline lme-miR161.2 & 21 & UCAAUGCAUUGAAAGUGACUA & scaffold 216 & $+/+$ & $5^{\prime}$ & 102 & -40.20 & 0.91 \\
\hline lme-miR162a & 22 & UGGAGGCAGCGGUUCAUCGAUC & scaffold 1363 & $+/+$ & $5^{\prime}$ & 116 & -37.20 & 0.77 \\
\hline lme-miR162a-3p & 21 & UCGAUAAACCUCUGCAUCCAG & scaffold 1363 & $+/+$ & $3^{\prime}$ & 131 & -44.30 & 0.76 \\
\hline lme-miR164a & 21 & UGGAGAAGCAGGGCACGUGCA & scaffold668 & $+/-$ & $5^{\prime}$ & 126 & -45.20 & 0.92 \\
\hline lme-miR164c & 21 & UGGAGAAGCAGGGCACGUGCG & scaffold369 & $+/+$ & $5^{\prime}$ & 83 & -38.90 & 0.97 \\
\hline lme-miR165a & 22 & UGGAGGCAGCGGUUCAUCGAUC & scaffold 1363 & $+/+$ & $5^{\prime}$ & 117 & -41.90 & 0.86 \\
\hline lme-miR165a-3p & 21 & UCGGACCAGGCUUCAUCCCCC & scaffold 830 & $+/+$ & $3^{\prime}$ & 125 & -45.40 & 1.05 \\
\hline lme-miR166a & 21 & GGACUGUUGUCUGGCUCGAGG & scaffold316 & $+/+$ & $5^{\prime}$ & 151 & -64.30 & 1.07 \\
\hline lme-miR166a-3p & 21 & UCGGACCAGGCUUCAUUCCCC & scaffold 774 & $+/+$ & $3^{\prime}$ & 92 & -32.30 & 0.73 \\
\hline lme-miR167a & 21 & UGAAGCUGCCAGCAUGAUCUA & scaffold 785 & $+/+$ & $5^{\prime}$ & 97 & -50.70 & 1.24 \\
\hline lme-miR167c & 21 & UAAGCUGCCAGCAUGAUCUUG & scaffold695 & $+/-$ & $5^{\prime}$ & 146 & -68.00 & 1.26 \\
\hline lme-miR 168a & 21 & UCGCUUGGUGCAGGUCGGGAA & scaffold 468 & $+1-$ & $5^{\prime}$ & 101 & -46.60 & 0.91 \\
\hline lme-miR 168a-3p & 21 & CCCGCCUUGCAUCAACUGAAU & scaffold369 & $+/+$ & $3^{\prime}$ & 101 & -47.40 & 0.89 \\
\hline lme-miR 169a & 21 & CAGCCAAGGAUGACUUGCCGA & scaffold191 & $+/+$ & $5^{\prime}$ & 227 & -67.80 & 1.09 \\
\hline lme-miR 169b & 21 & CAGCCAAGGAUGACUUGCCGG & scaffold 872 & $+/+$ & $5^{\prime}$ & 216 & -59.50 & 0.94 \\
\hline lme-miR 169d & 21 & UGAGCCAAGGAUGACUUGCCG & scaffold792 & $+/+$ & $5^{\prime}$ & 121 & -45.90 & 0.90 \\
\hline lme-miR $169 \mathrm{~h}$ & 21 & UAGCCAAGGAUGACUUGCCUG & scaffold 1032 & $+/+$ & $5^{\prime}$ & 131 & -43.30 & 0.92 \\
\hline lme-miR 169b-3p & 22 & GGCAAGUUGUCCUUCGGCUACA & scaffold 467 & $+/+$ & $3^{\prime}$ & 112 & -58.60 & 1.43 \\
\hline lme-miR miR170 & 21 & UAUUGGCCUGGUUCACUCAGA & scaffold 1132 & $+/+$ & $5^{\prime}$ & 83 & -33.60 & 0.96 \\
\hline lme-miR 170-3p & 21 & UGAUUGAGCCGUGUCAAUAUC & scaffold 1132 & $+/-$ & $3^{\prime}$ & 81 & -27.40 & 0.78 \\
\hline lme-miR 171b & 21 & AGAUAUUAGUGCGGUUCAAUC & scaffold 490 & $+/-$ & $5^{\prime}$ & 91 & -36.80 & 0.99 \\
\hline lme-miR 171a-3p & 21 & UGAUUGAGCCGCGCCAAUAUC & scaffold299 & $+/+$ & $3^{\prime}$ & 85 & -33.60 & 0.93 \\
\hline lme-miR 171b-3p & 21 & UUGAGCCGUGCCAAUAUCACG & scaffold 433 & $+/+$ & $3^{\prime}$ & 96 & -40.30 & 1.01 \\
\hline lme-miR $172 \mathrm{a}$ & 21 & AGAAUCUUGAUGAUGCUGCAU & scaffold 981 & $+/+$ & $3^{\prime}$ & 105 & -35.90 & 1.12 \\
\hline lme-miR $172 \mathrm{c}$ & 21 & AGAAUCUUGAUGAUGCUGCAG & scaffold 997 & $+/+$ & $3^{\prime}$ & 106 & -40.70 & 1.13 \\
\hline lme-miR 319a & 21 & UUGGACUGAAGGGAGCUCCCU & scaffold 1047 & $+/+$ & $3^{\prime}$ & 181 & -76.20 & 1.14 \\
\hline lme-miR 319c & 21 & UUGGACUGAAGGGAGCUCCUU & scaffold 488 & $+/-$ & $3^{\prime}$ & 173 & -69.60 & 0.98 \\
\hline lme-miR 390a & 21 & AAGCUCAGGAGGGAUAGCGCC & scaffold 237 & $+/+$ & $5^{\prime}$ & 91 & -43.40 & 1.11 \\
\hline lme-miR 391 & 21 & UUCGCAGGAGAGAUAGCGCCA & scaffold 432 & $+/+$ & $5^{\prime}$ & 82 & -34.80 & 0.87 \\
\hline lme-miR 391-3p & 21 & ACGGUAUCUCUCCUACGUAGC & scaffold 432 & $+/+$ & $3^{\prime}$ & 87 & -36.30 & 0.84 \\
\hline lme-miR 393a & 22 & UCCAAAGGGAUCGCAUUGAUCC & scaffold 997 & $+/+$ & $5^{\prime}$ & 156 & -53.90 & 1.00 \\
\hline lme-miR 393b-3p & 21 & AUCAUGCGAUCUCUUUGGAUU & scaffold 997 & $+/+$ & $3^{\prime}$ & 141 & -50.90 & 1.04 \\
\hline lme-miR 394a & 20 & UUGGCAUUCUGUCCACCUCC & scaffold 420 & $+/+$ & $5^{\prime}$ & 126 & -47.00 & 0.85 \\
\hline lme-miR 394b-3p & 21 & AGGUGGGCAUACUGCCAAUAG & scaffold 356 & $+/+$ & $3^{\prime}$ & 111 & -48.70 & 1.08 \\
\hline lme-miR 395a & 21 & CUGAAGUGUUUGGGGGAACUC & scaffold 904 & $+/-$ & $3^{\prime}$ & 92 & -44.30 & 1.10 \\
\hline lme-miR 395b & 21 & CUGAAGUGUUUGGGGGGACUC & scaffold 904 & $+/+$ & $3^{\prime}$ & 106 & -35.90 & 1.00 \\
\hline lme-miR 396a & 21 & UUCCACAGCUUUCUUGAACUG & scaffold339 & $+/-$ & $5^{\prime}$ & 106 & -34.50 & 0.88 \\
\hline
\end{tabular}


Table 1 continued

\begin{tabular}{|c|c|c|c|c|c|c|c|c|}
\hline Identified miRNAs & LM (nt) & miRNA sequences & Input sequence & Strand & Location & LP (nt) & $\operatorname{MFEs}(\Delta G)$ & MFEI \\
\hline lme-miR 396b & 21 & UUCCACAGCUUUCUUGAACUU & scaffold 127 & $+/+$ & $5^{\prime}$ & 132 & -43.70 & 1.15 \\
\hline lme-miR 396a-3p & 21 & GUUCAAUAAAGCUGUGGGAAG & scaffold 306 & $+/+$ & $3^{\prime}$ & 117 & -41.30 & 0.96 \\
\hline lme-miR 396b-3p & 21 & GCUCAAGAAAGCUGUGGGAAA & scaffold 127 & $+/+$ & $3^{\prime}$ & 156 & -55.50 & 1.13 \\
\hline lme-miR 397a & 21 & UCAUUGAGUGCAGCGUUGAUG & scaffold 299 & $+1-$ & $5^{\prime}$ & 96 & -26.80 & 0.96 \\
\hline lme-miR 398a-3p & 21 & UGUGUUCUCAGGUCACCCCUU & scaffold 302 & $+/+$ & $3^{\prime}$ & 101 & -42.90 & 1.13 \\
\hline lme-miR 398b-3p & 21 & UGUGUUCUCAGGUCACCCCUG & scaffold534 & $+1-$ & $3^{\prime}$ & 115 & -42.80 & 0.82 \\
\hline lme-miR 399a & 21 & UGCCAAAGGAGAUUUGCCCUG & scaffold 904 & $+1-$ & $3^{\prime}$ & 126 & -41.60 & 0.97 \\
\hline lme-miR 399b & 21 & UGCCAAAGGAGAGUUGCCCUG & scaffold 971 & $+1-$ & $3^{\prime}$ & 146 & -51.80 & 1.01 \\
\hline lme-miR 399c-3p & 21 & UGCCAAAGGAGAGUUGCCCUG & scaffold971 & $+1-$ & $3^{\prime}$ & 141 & -51.20 & 1.04 \\
\hline lme-miR 408 & 21 & ACAGGGAACAAGCAGAGCAUG & scaffold613 & $+/+$ & $5^{\prime}$ & 96 & -39.50 & 0.90 \\
\hline lme-miR 408-3p & 21 & AUGCACUGCCUCUUCCCUGGC & scaffold613 & $+1-$ & $3^{\prime}$ & 101 & -39.60 & 0.84 \\
\hline lme-miR 828 & 22 & UCUUGCUUAAAUGAGUAUUCCA & scaffold 236 & $+/+$ & $5^{\prime}$ & 101 & -35.80 & 1.02 \\
\hline lme-miR 2111a & 21 & UAAUCUGCAUCCUGAGGUUUA & scaffold668 & $+/+$ & $5^{\prime}$ & 101 & -38.30 & 1.18 \\
\hline lme-miR 2111b-3p & 21 & AUCCUCGGGAUACAGUUUACC & scaffold602 & $+/+$ & $3^{\prime}$ & 76 & -29.90 & 1.11 \\
\hline
\end{tabular}

$L M$ length of mature miRNAs, $L P$ length of precursor

precursors with higher MFEI values is presented in Fig. 1 (Top 20). Previous reports suggested that uracil at the first position of the sequence of miRNA play an important role in miRNA-mediated regulations in plant (Zhang et al. 2008). In this study, uracil was observed to be predominant at the first position of $\sim 60 \%$ mature miRNA sequences.

\section{Experimental validation of putative maca miRNAs}

The efficiency of the computational strategy was further verified by RT-PCR based experimental procedure. The randomly selected four miRNAs lme-miR160a, lmemiR164c, lme-miR 166a, and lme-miR 319a from maca
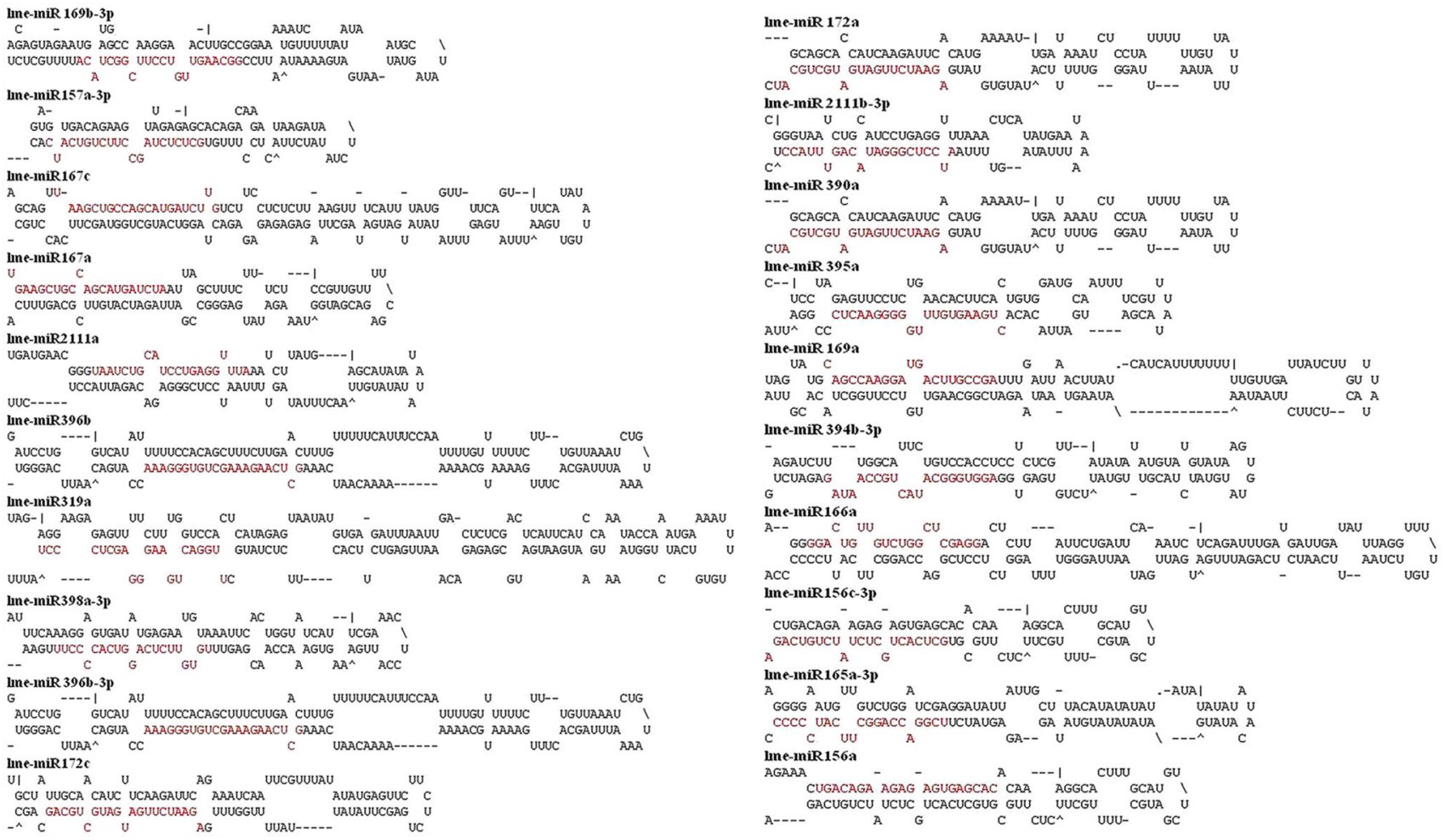

Fig. 1 Secondary structure (stem-loop) of the maca miRNA precursors with higher MFEI values (Top 20). Respective miRNAs are represented with red font 
were subjected to validation studies. All these maca miRNAs showed confirmation through experimental validation (Fig. 2).

\section{Potential targets of putative maca miRNAs and their function}

A total of 99 potential targets were identified and most of them were functionally categorized as transcription factors. Important transcription factors targeted by maca miRNAs include Squamosa promoter-binding protein/SPB (miR156/ 157), Auxin-responsive factor (miR160), Cytochrome P-450 (miR162), NAM protein (miR164), Class III HD-Zip (miR165/166), MYB (miR172/319), F-box protein (miR394/399) (Table 2). These transcription factors are known to play a role in metabolic processes and stress response signaling in plants. Moreover, to improve the efficient understanding of miRNA regulation in maca, gene ontology analysis of the identified target transcript was executed by AmiGo (http://amigo.geneontology.org/ amigo), and high involvement of the target transcripts in the biological, molecular, and cellular process was observed (Fig. 3).

Surrounding environment is the key factor for proper growth and development of plants. Stress-sensitive plants often show limited growth during environmental stresses while stress-tolerant plants employ several complex defense mechanisms including miRNA-mediated posttranscriptional gene silencing (Sunkar et al. 2007). Although few discrete studies have been performed to check the alterations of miRNAs during cold and irradiance stresses in tolerant plants, the exact molecular mechanism is still unclear. Zhang et al. (2014) reported that during cold stress 31 miRNAs were up-regulated and 43 were downregulated in cold tolerant tea variety 'Yingshuang' while 46 miRNAs were up-regulated and 45 down-regulated in

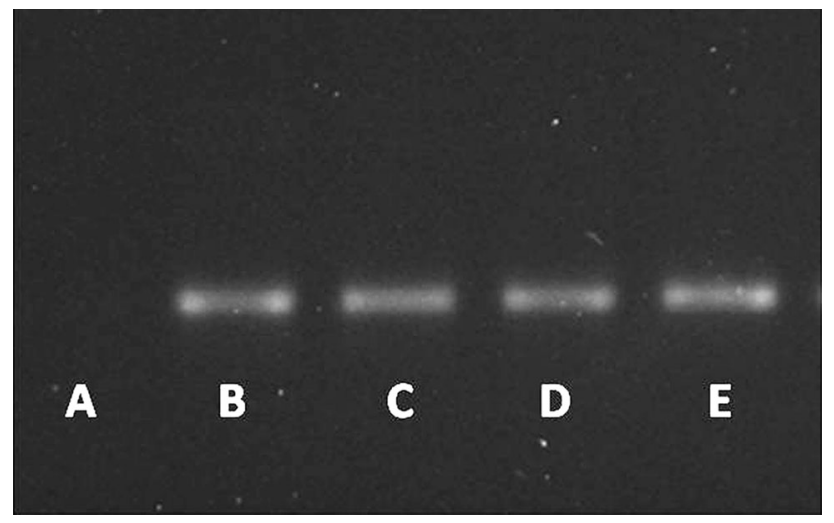

Fig. 2 Validation of some maca miRNAs by RT-PCR. The resulting PCR products are checked in $2 \%$ agarose gel with EtBr staining. a Negative control, b lme-miR160a, c lme-miR164c, d lme-miR 166a and e lme-miR 319a
Table 2 Potential targets of identified maca miRNAs

\begin{tabular}{|c|c|}
\hline miRNA & Targeted proteins (number) \\
\hline $156 / 157$ & Squamosa promoter-binding-like protein (24) \\
\hline \multirow[t]{3}{*}{159} & MYB (4) \\
\hline & Pectin acetylesterase (1) \\
\hline & Pyruvate orthophosphate dikinase (5) \\
\hline 160 & Auxin response factor (4) \\
\hline 162 & Cytochrome P450-like protein (1) \\
\hline \multirow[t]{2}{*}{164} & CUP-SHAPED COTYLEDON/CUC (3) \\
\hline & NAM protein-like (6) \\
\hline 165 & HD-Zip protein (2) \\
\hline 166 & HD-Zip protein (2) \\
\hline 168 & Glutathione transferase (1) \\
\hline \multirow[t]{2}{*}{171} & Scarecrow-like protein (2) \\
\hline & Protein kinase (2) \\
\hline \multirow[t]{2}{*}{172} & APETALA 2 (8) \\
\hline & MYB (2) \\
\hline 319 & MYB (3) \\
\hline \multirow[t]{2}{*}{390} & Protein kinase (1) \\
\hline & Glutamate dehydrogenase (1) \\
\hline 391 & Protein kinase (2) \\
\hline 393 & WRKY transcription factor (1) \\
\hline \multirow[t]{3}{*}{394} & F-box only protein (1) \\
\hline & Lipase-like protein (1) \\
\hline & Loricrin-like protein (1) \\
\hline \multirow[t]{2}{*}{395} & ATP sulfurylase (9) \\
\hline & Transcription factor bZIP (2) \\
\hline 397 & Laccase precursor (3) \\
\hline \multirow[t]{2}{*}{399} & F-box protein (1) \\
\hline & Cytochrome P450-like protein (1) \\
\hline 408 & Ethylene-responsive transcription factor (1) \\
\hline 828 & Kinesin $2(2)$ \\
\hline 2111 & Flavanone 3-hydroxylase-like protein (2) \\
\hline
\end{tabular}

sensitive variety 'Baiye 1'. Casadevall et al. (2013) showed that up-regulation of miR396 enhances survival of Arabidopsis thaliana under UV-B radiation. Nevertheless, regulation of plant miRNAs at high-altitude environment (combined effect of extreme cold, strong wind, and oxidizing air pollutants) has been poorly studied and hence maca could provide new insights into the understanding of stress-responsive miRNAs at higher altitude. On the other hand, few workers also reported that miRNAs can influence the production of bioactive compounds/secondary metabolites in the medicinal plants (Robert-Seilaniantz et al. 2011; Singh et al. 2016). Nonetheless, identification of miRNAs and their targets is the key step to initiate a miRNA-related study in a plant. This study can be of immensely helpful for future research on miRNA-mediated 


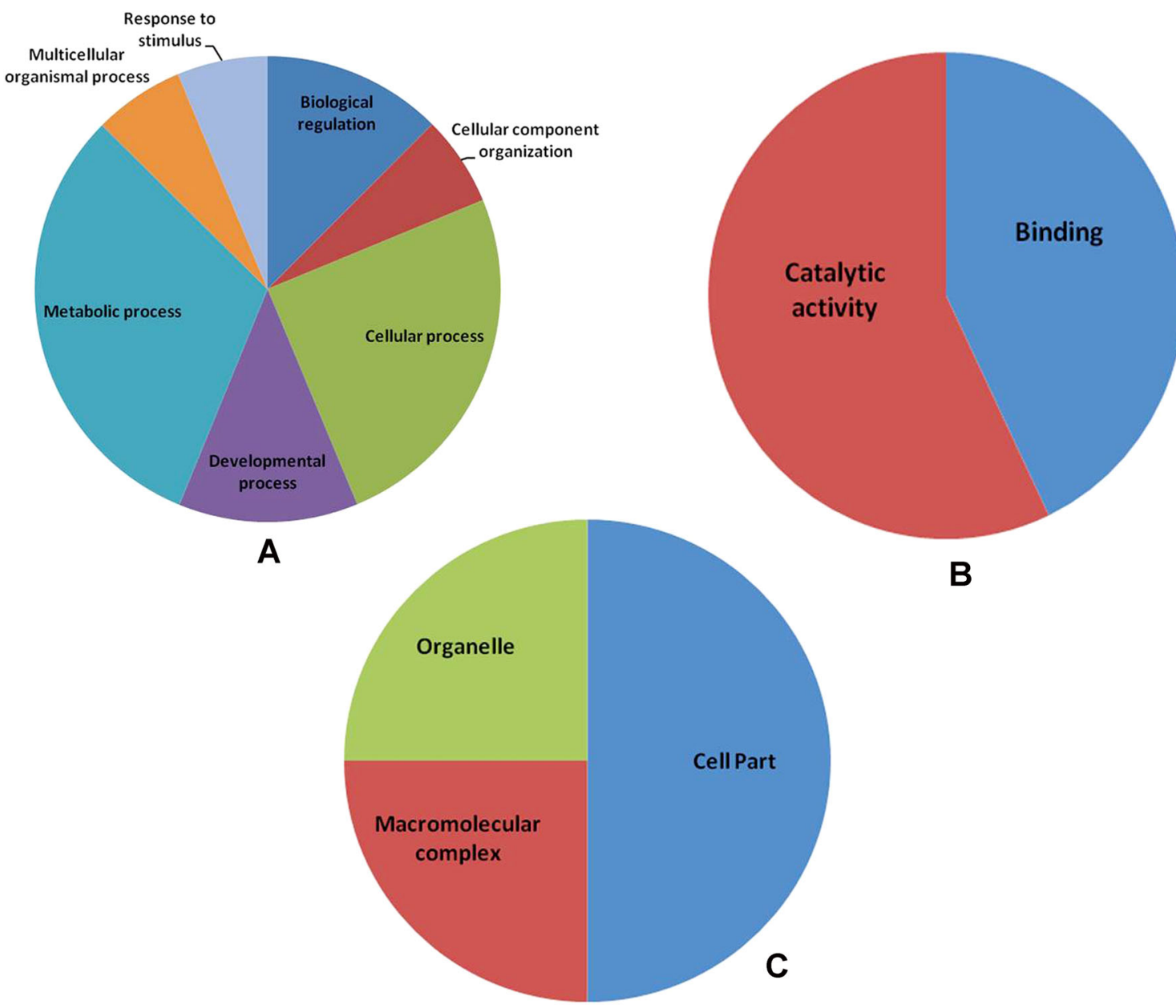

Fig. 3 GO analysis of target transcripts regulated by identified miRNAs: a biological process, $\mathbf{b}$ molecular function and $\mathbf{c}$ cellular component

stress response signaling as well as the production of bioactive compounds/secondary metabolites in medicinal plants.

\section{Conclusion}

In this study a total of 62 conserved miRNAs belonging to 28 families were first time identified in a high-altitude plant such as maca. To validate the expression of potential miRNAs in maca, a RT-PCR approach was performed and 4 miRNA families were detected. Moreover, a total of 63 potential targets were predicted and they were found to be involved in development, metabolism and stress responses.

Acknowledgements Author is thankful to Prof. Amita Pal, Bose Institute for valuable guidance and help.

\section{Compliance with ethical standards}

Conflict of interest The author declares that he has no conflict of interest.

\section{References}

Casadevall R, Rodriguez RE, Debernardi JM et al (2013) Repression of growth regulating factors by the microRNA396 inhibits cell proliferation by UV-B radiation in Arabidopsis leaves. Plant Cell 25:3570-3583. doi:10.1105/tpc.113.117473

Fukudome A, Fukuhara T (2017) Plant dicer-like proteins: doublestranded RNA-cleaving enzymes for small RNA biogenesis. J Plant Res 130:33-34. doi:10.1007/s10265-016-0877-1

Huang Y, Cheng J, Luo F et al (2016) Genome-wide identification and characterization of microRNA genes and their targets in large yellow croaker (Larimichthys crocea). Gene 576:261-267. doi:10.1016/j.gene.2015.10.044

Khraiwesh B, Zhu J-K, Zhu J (2012) Role of miRNAs and siRNAs in biotic and abiotic stress responses of plants. Biochim Biophys Acta Gene Regul Mech 1819:137-148. doi:10.1016/j.bbagrm. 2011.05.001

Kundu A, Paul S, Dey A, Pal A (2017) High throughput sequencing reveals modulation of microRNAs in Vigna mungo upon Mungbean Yellow Mosaic India Virus inoculation highlighting stress regulation. Plant Sci 257:96-105. doi:10.1016/j.plantsci. 2017.01.016

Naya L, Paul S, Valdés-López O et al (2014) Regulation of copper homeostasis and biotic interactions by microRNA 398b in common bean. PLoS One. doi:10.1371/journal.pone.0084416 
Paul S, Kundu A, Pal A (2011) Identification and validation of conserved microRNAs along with their differential expression in roots of Vigna unguiculata grown under salt stress. Plant Cell Tissue Organ Cult 105:233-242. doi:10.1007/s11240-010-98577

Piacente S, Carbone V, Plaza A et al (2002) Investigation of the tuber constituents of maca (Lepidium meyenii Walp). J Agric Food Chem 50:5621-5625. doi:10.1021/jf020280x

Robert-Seilaniantz A, MacLean D, Jikumaru Y et al (2011) The microRNA miR393 re-directs secondary metabolite biosynthesis away from camalexin and towards glucosinolates. Plant $\mathbf{J}$ 67:218-231. doi:10.1111/j.1365-313X.2011.04591.x

Shin B-C, Lee MS, Yang EJ et al (2010) Maca (L. meyenii) for improving sexual function: a systematic review. BMC Complement Alt Med 10:44. doi:10.1186/1472-6882-10-44

Singh N, Srivastava S, Sharma A (2016) Identification and analysis of miRNAs and their targets in ginger using bioinformatics approach. Gene 575:570-576. doi:10.1016/j.gene.2015.09.036

Sunkar R, Chinnusamy V, Zhu J, Zhu JK (2007) Small RNAs as big players in plant abiotic stress responses and nutrient deprivation. Trends Plant Sci 12:301-309

Wang L, Liu H, Li D, Chen H (2011) Identification and characterization of maize microRNAs involved in the very early stage of seed germination. BMC Gen 12:154. doi:10.1186/1471-2164-12154

Wang M, Wang Q, Wang B (2012) Identification and characterization of microRNAs in asiatic cotton (Gossypium arboreum L.). PLoS One. doi:10.1371/journal.pone.0033696

Yang W, Liu X, Zhang J et al (2010) Prediction and validation of conservative microRNAs of Solanum tuberosum L. Mol Biol Rep 37:3081-3087. doi:10.1007/s11033-009-9881-z

Zhang B, Pan X, Cobb GP, Anderson TA (2006) Plant microRNA: a small regulatory molecule with big impact. Dev Biol 289:3-16

Zhang B, Pan X, Stellwag EJ (2008) Identification of soybean microRNAs and their targets. Planta 229:161-182. doi:10.1007/ s00425-008-0818-x

Zhang Y, Zhu X, Chen X et al (2014) Identification and characterization of cold-responsive microRNAs in tea plant (Camellia sinensis) and their targets using high-throughput sequencing and degradome analysis. BMC Plant Biol 14:271. doi:10.1186/ s12870-014-0271-x

Zhang J, Tian Y, Yan L et al (2016) Genome of plant maca (Lepidium meyenii) illuminates genomic basis for high-altitude adaptation in the central Andes. Mol Plant 9:1066-1077. doi:10.1016/j. molp.2016.04.016 